\title{
Empleo de tecnologías de geomática para la evaluación de riesgos de inundaciones como consecuencia del cambio climático y las afectaciones a la fitodiversidad
}

\author{
Employment of geomatics technologies for the \\ evaluation of risks of floods as consequence of the \\ climatic change and the affectations to phytodiversity
}

Raimundo Márquez Gurri'

Nicolás Quintana Bernabé

Marta Margarita Pérez Rojas ${ }^{3}$

Freddy Araya Rodríguez ${ }^{4}$

Fecha de recepción: 20 de enero del 2012

Fecha de aprobación: 19 de marzo del 2012

Márquez, R; Quintana, N; Pérez, Marta; Araya.

F. Empleo de tecnologías de geomática para

la evaluación de riesgos de inundaciones

como consecuencia del cambio climático y las

afectaciones a la fitodiversidad. Tecnología en

Marcha. Vol. 25, № 4. Pág 76-80

I Ingeniero aerofotogeodesta. Universidad de Ciego de Ávila,

Departamento Informática-Geodinámica, Escuela Informática

Cuba.

Correo electrónico: rmarquezg@ingenieria.unica.cu

2 Estadístico-matemático. Universidad de Ciego de Ávila, Departamento Informática-Geodinámica, Escuela Informática Cuba. Correo electrónico: nquintana@informatica.unica.cu

3 Especialista de Geomática. Agencia Geocuba Ciego de Ávila, Empresa Geocuba Camaguey-Ciego de Ávila, Cuba. Correo electrónico: mperez@informatica.unica.cu

4 Doctor en Ciencias Agrícolas. Tecnológico de Costa Rica, Sede San Carlos. Teléfono: 240 I-3I40

Correo electrónico: faraya@itcr.ac.cr 


\section{Palabras clave}

MDT, SIG, escurrimiento superficial, cambios climáticos globales.

\section{Resumen}

El cambio climático global que tiene lugar en los tiempos actuales tiene diversos orígenes, provocando no pocas polémicas y declaraciones provenientes de diferentes áreas del conocimiento. Las inundaciones son parte de las consecuencias de los fenómenos que ocurren en momentos en que las crisis se acentúan y el medio ambiente se deteriora.

En este trabajo se presentan algunas experiencias en el empleo de las herramientas geomáticas para la minimización de riesgos, ante la posibilidad de la ocurrencia de eventos extraordinarios: fuertes lluvias en cortos periodos de tiempo e inundaciones, como consecuencia del cambio climático, particularizando en las vías de comunicación como elementos antrópicos, la interrupción del curso natural de las aguas sobre el relieve topográfico y los impactos sobre la fitodiversidad que acontecen como resultado de las mismas.

\section{Key words}

DTM, GIS, run-off surface, global climate change.

\section{Abstract}

The global climatic change that takes place in the current times has diverse origins, not causing few polemics and declarations from different areas of knowledge. The floods are part of the consequences of the phenomena that take place when crisis are accentuated and the environment deteriorates.

This research presents some experiences in the employment of the geomatics tools for the minimization of risks when there is the possibility of the occurrence of extraordinary events: strong rains in short periods of time and floods, as a consequence of the climatic change, particularizing in the communication roads, as antropics elements and the interruption of the natural course of the waters on the topographical relief and the impacts on phitodiversity.

\section{Introducción}

El ser humano forma parte del mundo vivo que lo rodea, a la vez que depende de él para la satisfacción de sus más perentorias e impostergables necesidades materiales y espirituales. La pérdida de recursos biológicos y su diversidad pone en peligro el suministro de alimentos, la provisión de madera, medicamentos y energía y las oportunidades para el recreo y el turismo.

Además, interfiere con las funciones ecológicas esenciales, tales como la regulación de la escorrentía, el control de la erosión del suelo, la asimilación de desechos, la purificación del agua y el ciclo del carbono y los nutrientes.

Es por ello que cada vez adquiere mayor urgencia la necesidad de salvaguardar estos recursos, pues el ritmo de pérdidas de lo que aún desconocemos es tal que si no se toman las medidas apropiadas nos veremos en un futuro no muy lejano abocados a una verdadera catástrofe.
Los tiempos actuales presuponen la introducción de nuevas tecnologías y conocimiento en casi todas las esferas de la vida, sin tecnocracias y con la participación de las comunidades de científicos y especialistas para garantizar su sostenibilidad.

Los datos geográficos en formato digital son un intento por modelar y describir el mundo real para su uso en análisis computarizados y despliegue de información gráfica.

Aunque consciente de que cualquier descripción del mundo real es una abstracción, parcial y única de las muchas posibles conceptualizaciones, de modo que no es una copia exacta de la realidad, ya que algunos elementos son aproximaciones, otros son simplificaciones y otros son ignorados, de manera que no hay datos totalmente "idénticos" a la realidad que tratan de describir; la era digital ha hecho numerosos aportes que han cambiado significativamente los conceptos básicos que los sustentan y no pocos se han modificado consecuentemente con la realidad. 
El escurrimiento superficial debe ser interpretado a partir de las más elaboradas técnicas matemáticas e informáticas, las cuales deben enfocarse en las consecuencias del exceso de la caída de agua pluvial y las razones por las que se hace necesario introducir nuevas herramientas que permitan mejorar la precisión de los análisis sobre la erosión de los suelos y la calidad de las aguas, entre otras, así como la simulación de su ocurrencia a diferentes porcentajes de probabilidades, con el fin de prevenir a los tomadores de decisión sobre las posibles pérdidas por su efecto.

Diversos autores han propuesto definiciones para la modelación digital del terreno, entre ellos Felicísimo (1994), quien las considera modelos simbólicos en los cuales las relaciones de correspondencia que se establecen con el objeto real tienen la forma de algoritmos o formalismos matemáticos.

Wegener (2000) e Ilwis (2003) lo plantean como una representación simplificada de un objeto de investigación, elaborado con la finalidad de descripción, explicación, previsión o planificación, abordándolo desde puntos de vista similares expresando para ello diferentes tipos y categorías. Consideramos que es imprescindible desarrollar un tratamiento de las tres dimensiones geométricas, añadiendo la altura (coordenada $z$ ) a las más habituales: coordenada $X$ y coordenada $Y$, y la consideración de otra referencia fundamental que es el "tiempo", que actúa realmente como una cuarta coordenada, necesaria para establecer la composición del elemento o fenómeno modelado en cada punto incluido en la base de datos.

En el presente trabajo se muestran los resultados de los estudios de la hidrología superficial de un territorio llano a partir de un Modelo Digital del Terreno (MDT) y su aprovechamiento efectivo en el entorno de un Sistema de Información Geográfica (SIG). También se estudia la ocurrencia de eventos pluviales extraordinarios, así como el escurrimiento superficial y sus efectos, entre otros.

Los SIG se empezaron a generalizar a partir de la década de 1980, pero su gestación y desarrollo se remontan dos décadas atrás, cuando, entre 1960 y 1964 se desarrolló el Canadian Geographic Information System (CGIS), con el objetivo de gestionar las informaciones sobre los bosques y superficies marginales de Canadá.
Bajo una estructura raster y vectorial que combinaba la cartografía con los datos necesarios para la gestión forestal, se realizaban estudios del volumen maderable, pistas de saca y además los informes de explotación para la administración forestal del país. Este sistema ha ido evolucionando y perfeccionándose, constituyendo una invaluable herramienta, según lo expuesto por Domínguez Bravo (2000).

Buena parte de los investigadores de estos laboratorios son los responsables del desarrollo y auge en los años 80 de los SIG entendidos como productos industriales. Es el momento del avance de los SIG vectoriales en el que se destaca la implantación de Arc/Info por ESRI (Earth Systems Research Institute). Domínguez Bravo, J. (2000).

Hoy día asistimos a la consolidación del SIG como industria, caracterizada por una progresiva integración de sistemas ráster y vectoriales y por el aumento de la importancia de las comunicaciones entre sistemas y la interface de usuario, así como por el uso de herramientas de programación de tipo "visual" basadas en la metodología "orientadas a objetos".

Los nuevos campos de innovación de los SIG son la integración en sistemas de soporte de decisiones, los llamados sistemas de sobremesa (divulgación de la cartografía y de la Información Geográfica), los sistemas y servidores de información geográfica en red y distribuidos (Internet) y los llamados SIG móviles (aplicación de los SIG en el ámbito de la telefonía móvil e integrados a la tecnología GPS (en sus variantes GPSMaps).

\section{Desarrollo}

La precipitación máxima probable para un intervalo de tiempo concreto se registra habitualmente para 24 horas, correspondientes al día pluviométrico, y se deduce a partir de los registros obtenidos por los pluviómetros o pluviógrafos ubicados cerca y en el interior de la cuenca. Con la ayuda de métodos estadísticos que emplean modelos de leyes de distribución de frecuencia, se pueden calcular los valores de precipitación máxima en los puntos de los que se dispone de información.

Tomando como referencia estos puntos de muestreo, y gracias a las técnicas de interpolación espacial que incorporan algunos softwares especializados y 
los SIG (p. ej. Kriging, Curvatura Mínima o el Inverso de la Distancia Pesada), es posible estimar estos valores en aquellos lugares que no cuentan con estas mediciones directas. Esta predicción matemática dota a la mencionada variable climática de continuidad en el espacio.

La representación de un MDT puede realizarse básicamente a través de dos estructuras de datos: la matriz regular de elevaciones (modelo raster) y una red de triángulos irregulares en el modelo vectorial (TIN ○ Triangulated Irregular Network) (Weibel \& Heller, 1991). Sin embargo, también se utilizan otras estructuras menos usuales, como las matrices jerárquicas escalables (quadtree en modelo raster), contornos (vectorial) y otras (Felicísimo, 1994).

En nuestro objeto de estudio empleamos como base las curvas de nivel que representan el relieve del mapa topográfico a escala 1: 10 000, con una equidistancia de I m y las cotas del terreno para la concepción del modelo; se incluyeron además los elementos de la hidrografía tales como: cotas hidrostáticas, embalses de mayor importancia, presas (cortina de embalse), ríos y arroyos, que aumentan la densificación y precisión del modelado de la superficie, incluyendo elementos (puentes, talud y cortes) de la planimetría, que aportan la información necesaria para poder realizar el tratamiento que lleva las curvas de nivel en estos lugares y tienen una interrelación directa con el relieve del terreno por modelar.

La vectorización se desarrolló de forma manual mediante el sistema AutoCAD Map 2005, hasta el intercambio a formato DXF, siendo estos los datos que posteriormente serán procesados con el objetivo de obtener un fichero ASCll con los datos correspondientes a los puntos de la malla, luego de someterse a un riguroso proceso de edición; los elementos necesarios se desarrollaron en función de la MET. 66-2.4 25:(2008).

Se comprobó la eficacia de la creación del relieve mediante técnicas de geoestadística, empleando un apropiado método de interpolación, como se expone en la siguiente figura:

Comúnmente, el parámetro establecido para describir la precisión de este tipo de modelos es el Error Medio Cuadrático (EMC), según Cebrián y Mark (1986), Weibel y Heller (1991) y Felicísimo (1994), que describe la variación global entre una serie de puntos medidos en el "mundo real" y sus correspondientes en el MDT. Este parámetro se establece de la manera siguiente:

$$
E M C=\sqrt{\frac{\sum_{i=1}^{\mu}\left(Z T_{i}-Z M D E_{i}\right)^{2}}{n}}
$$

donde: $Z T_{i}=$ altitud medida sobre el terreno con gran precisión en el punto,$Z M D E_{i}=$ altitud medida sobre MDT en el punto $;$ y $n=$ número de puntos muestrales.

El cálculo de dicho parámetro se estableció realizando una comparación entre una serie de puntos con
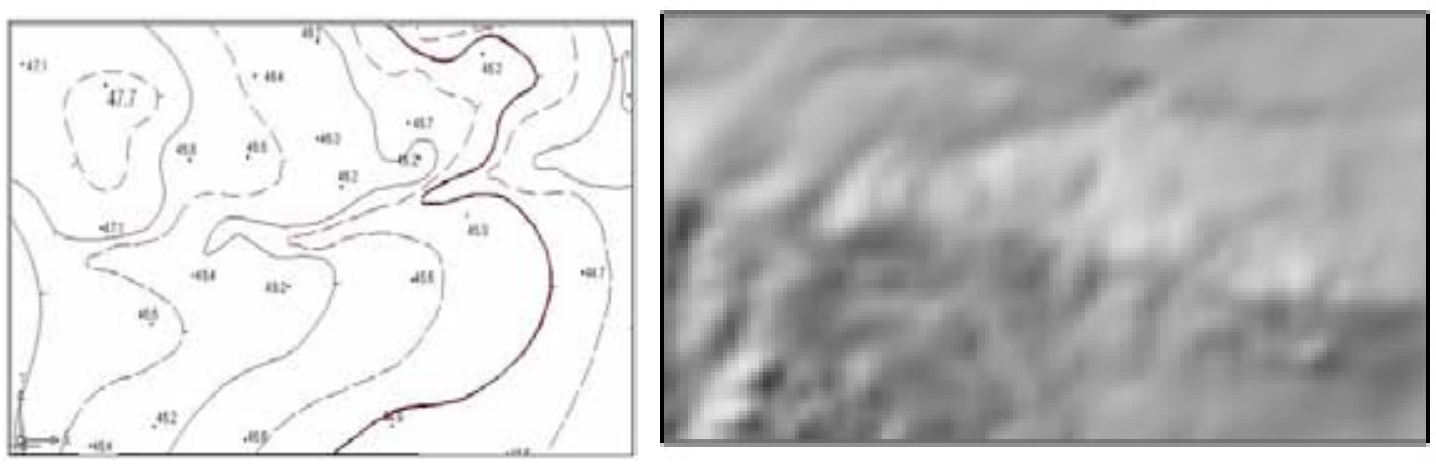

(a) (b)

Figura I. (a) Relieve digital creado tomando como base el mapa topográfico vectorial.

(b) Imagen generada de la interpolación geoestadística de las cotas del relieve. 
altitudes conocidas, los cuales pueden ser verificados con sus homólogos en el MDT.

Evaluar el comportamiento de una cuenca o subcuenca hidrológica con la ayuda de un modelo matemático cobra sentido cuando la información resultante obtenida con el mismo es imprescindible para lograr un objetivo difícilmente alcanzable por otros medios menos costosos, lo que según Triviño y Ortiz (2004) constituye un argumento que justifica el estudio hidrogeomorfológico, tales como los análisis de riesgo de inundaciones, con un amplio efecto en su interacción con elementos antrópicos como diques, carreteras y caminos.

Los modelos facilitan la manipulación de las variables que intervienen en el proceso de conversión lluvia-caudal, aportando datos sobre la distribución espacial del escurrimiento superficial y mejorando la calidad de la cartografía temática sobre zonas inundables y zonas con riesgo de inundación.

El campo de aplicaciones de los MDT es extenso, siendo además una herramienta que se incorpora con facilidad a distintos fines de carácter ambiental. Sircar y Cebrián (1990) y Cebrián y Mark (1986) realizaron un compendio de las principales aplicaciones de los MDT, entre las cuales destacan las ciencias de la tierra, para facilitar datos usados en el estudio del relieve, pendientes, geoformas, la definición de cuencas fluviales y redes de drenaje y la correlación entre técnicas de explotación en la erosión del suelo y en la contaminación de las aguas.

Se debe añadir a Weibel y Heller (|99|), quienes destacan la importancia de los MDT para la realización de estudios de impacto ambiental, localización industrial y estudios de capacidad de cultivos y de nuestros resultados la aplicación en los estudios de vulnerabilidad y riesgos de inundaciones ante eventos pluviométricos extraordinarios, como efecto de la interacción del relieve natural con elementos antrópicos.

\section{Conclusiones}

La integración de los MDT al mundo de los SIG ofrece nuevas aplicaciones y diferentes opciones de utilización, como es la generación de nuevas informaciones espaciales a partir de ellos, tales como pendiente, orientación, efectos de iluminación, entre otros, ofreciendo diferentes formas fisiográficas que nos permiten verificar la capacidad del procedimien- to planteado para la definición del sentido y dirección de flujo del escurrimiento superficial. Subraya la importancia de establecer una apropiada integración de las relaciones de los modelos de datos presentes en los atributos principales de los MDT (altura y tiempo), mediante una mayor fundamentación teórica en el campo de los modelos de datos.

\section{Bibliografía}

Barredo, J.I. \& Bosque, J. (1996). Delimitación de unidades homogéneas del relieve a partir de un modelo digital de elevaciones. Estudios geográficos, No. 225, pp. 615643.

Cebrián, J. \& Mark, D. (1986). Modelos topográficos digitales. Métodos Cuantitativos en Geografía: Enseñanza, investigación y planeamiento. Madrid: AGE.

Domínguez, B.J. (2000). Breve Introducción a la Cartografía y a los Sistemas de Información Geográfica. CIEMAT. Madrid: Ministerio de Ciencia y Tecnología. ISSN: I | 35-9420 38 pp.

Felicísimo, A.M. (1994). Modelos digitales del terreno: principios y aplicaciones en las ciencias ambientales. Oviedo, España: Pentalfa Ediciones. 222 p. ISBN: 84-7848-475-2

IIwis 3.0. User's Guide. Chapter 10. Using Digital Elevation Models, pp. 387 (2003).

MET. 66-2.4 25: Metodología general para la confección de Modelos Digitales del Terreno (MDT) a partir de bases cartográficas digitales, aplicados a la ortorrectificación de imágenes aeroespaciales. Pp. 4-27. La Habana: Geocuba (2008)

Olaya, V. (2004). Hidrología Computacional y Modelos Digitales del Terreno -Teoría, práctica y filosofía de una nueva forma de análisis hidrológico. Obtenido desde: http://heart.sourceforge.net/sp/index.htm). [Consulta: febrero, 12/2007].

Sircar, J. \& Cebrián, J. (1990). Creación de modelos topográficos digitales (MTD) a partir de curvas de nivel rasterizadas. Anales de Geografía de la Universidad Complutense, No. 10.

Triviño, A. \& Ortiz, S. (2004). Metodología para la modelación distribuida de la escorrentía superficial y la delimitación de zonas inundables en ramblas y ríosrambla mediterráneos. Investigaciones Geográficas No. 35, pp 67-83.

Wegener, M. (2000). Spatial Models and SIG. London: Taylor and Francis.

Weibel, R. \& Heller, M. (|99|). Digital terrain model. Geographical Information Systems, Vol. I, Longman, Scientific and Technical. 